\title{
The cortex-based alignment approach to TMS coil positioning
}

Citation for published version (APA):

Duecker, F., Frost, M. A., de Graaf, T. A., Gräwe, B., Jacobs, C., Goebel, R. W., \& Sack, A. T. (2014). The cortex-based alignment approach to TMS coil positioning. Journal of Cognitive Neuroscience, 26(10), 2321-2329. https://doi.org/10.1162/jocn_a_00635

Document status and date:

Published: 04/04/2014

DOI:

10.1162/jocn_a_00635

Document Version:

Publisher's PDF, also known as Version of record

Document license:

Taverne

Please check the document version of this publication:

- A submitted manuscript is the version of the article upon submission and before peer-review. There can be important differences between the submitted version and the official published version of record.

People interested in the research are advised to contact the author for the final version of the publication, or visit the DOI to the publisher's website.

- The final author version and the galley proof are versions of the publication after peer review.

- The final published version features the final layout of the paper including the volume, issue and page numbers.

Link to publication

\footnotetext{
General rights rights.

- You may freely distribute the URL identifying the publication in the public portal. please follow below link for the End User Agreement:

www.umlib.nl/taverne-license

Take down policy

If you believe that this document breaches copyright please contact us at:

repository@maastrichtuniversity.nl

providing details and we will investigate your claim.
}

Copyright and moral rights for the publications made accessible in the public portal are retained by the authors and/or other copyright owners and it is a condition of accessing publications that users recognise and abide by the legal requirements associated with these

- Users may download and print one copy of any publication from the public portal for the purpose of private study or research.

- You may not further distribute the material or use it for any profit-making activity or commercial gain

If the publication is distributed under the terms of Article $25 \mathrm{fa}$ of the Dutch Copyright Act, indicated by the "Taverne" license above, 


\title{
The Cortex-based Alignment Approach to TMS Coil Positioning
}

\author{
Felix Duecker, Martin A. Frost, Tom A. de Graaf, Britta Graewe, \\ Christianne Jacobs, Rainer Goebel, and Alexander T. Sack
}

\begin{abstract}
TMS allows noninvasive manipulation of brain activity in healthy participants and patients. The effectiveness of TMS experiments critically depends on precise TMS coil positioning, which is best for most brain areas when a frameless stereotactic system is used to target activation foci based on individual fMRI data. From a purely scientific perspective, individual fMRI-guided TMS is thus the method of choice to ensure optimal TMS efficiency. Yet, from a more practical perspective, such individual functional data are not always available, and therefore alternative TMS coil positioning approaches are often applied, for example, based on functional group data reported in Talairach coordinates. We here propose a novel method for TMS coil positioning that is based on functional group data, yet only requires individual anatomical data. We used cortex-based alignment (CBA) to transform individual anatomical data to an atlas brain that includes probabilistic group maps of two functional regions (FEF and hMT+/V5). Then, these functional group maps were
\end{abstract}

\section{INTRODUCTION}

TMS is a noninvasive brain interference technique that is widely used to investigate brain-behavior relationships in the healthy and diseased human brain (Sack, 2006; Hallett, 2000; Pascual-Leone, Walsh, \& Rothwell, 2000; Walsh \& Cowey, 2000). TMS can provide excellent temporal resolution because of the brevity of a single TMS pulse (less than $1 \mathrm{msec}$ ) and good spatial resolution. The spatial resolution of TMS critically depends on the TMS coil design (Deng, Lisanby, \& Peterchev, 2012). In the majority of studies, the so-called figure-of-eight coils are used that are purpose-built to generate a very focal magnetic field and thus allow selective stimulation of individual functional brain areas. This spatial specificity of TMS is very desirable in most cases and is paramount for investigating "causal" structure-function relationships. However, effective spatial resolution depends not only on coil design but also on the accuracy of TMS coil positioning. In fact, the more spatially specific TMS is, the more important it becomes to ensure that TMS is applied exactly to the brain location of interest.

Maastricht University back-transformed to the individual brain anatomy, preserving functional-anatomical correspondence. As a proof of principle, the resulting CBA-based functional targets in individual brain space were compared with individual FEF and hMT+/V5 hotspots as conventionally localized with individual fMRI data and with targets based on Talairach coordinates as commonly done in TMS research in case only individual anatomical data are available. The CBA-based approach significantly improved localization of functional brain areas compared with traditional Talairach-based targeting. Given the widespread availability of CBA schemes and preexisting functional group data, the proposed procedure is easy to implement and at no additional measurement costs. However, the accuracy of individual fMRIguided TMS remains unparalleled, and the CBA-based approach should only be the method of choice when individual functional data cannot be obtained or experimental factors argue against it.

Different approaches for TMS coil positioning have been developed over the years (Sack et al., 2009; Sparing, Buelte, Meister, Paus, \& Fink, 2008). In some cases, the immediate consequences of a single TMS pulse can be used as an index for effective stimulation. By moving along primary motor cortex, one can induce muscle twitches that correspond to the organization of the motor "homunculus," allowing identification of the optimal TMS coil position to target the intended motor representation (e.g., Wassermann, Mcshane, Hallett, \& Cohen, 1992). Similarly, TMS over early visual cortex induces phosphenes that correspond to the retinotopic organization of visual cortex (e.g., Kammer, Puls, Erb, \& Grodd, 2005). When the TMS coil is positioned based on such functional markers, the resulting TMS target points have been shown to closely match localization based on individual fMRI data and may be considered optimal (Salminen-Vaparanta, Noreika, Revonsuo, Koivisto, \& Vanni, 2012; Thielscher, Reichenbach, Uğurbil, \& Uludağ, 2010; Thompson, Aaen-Stockdale, Koski, \& Hess, 2009). However, the majority of brain areas are "silent," that is, a TMS pulse does not produce a readily observable response, thus requiring alternative TMS coil positioning approaches. In early work, the TMS coil was positioned relative to functional markers as described 
above or simply shifted several centimeters from cranial landmarks like the inion. Another popular method was to borrow the international 10-20 coordinate system from EEG research (Jasper, 1958), which assumes that certain electrode positions correspond to certain anatomical regions (see Herwig, Satrapi, \& Schönfeldt-Lecuona, 2003). But as the availability of imaging tools increased, "neuronavigation" by a frameless stereotactic system became a preferred method overcoming various limitations of previous approaches. Both the TMS coil and the participant's head are tracked and coregistered in space, allowing precise (online) targeting of a predefined brain area (Cohen Kadosh et al., 2007; Sack, Kohler, Linden, Goebel, \& Muckli, 2006; Schönfeldt-Lecuona et al., 2005; Herwig et al., 2002; Paus, 1999).

This brain area can be defined in several ways. First, one might determine the TMS "target point" based on individual anatomical data. For instance, one could inspect the anatomical data and manually define the inferior parietal sulcus. For many brain areas, this approach is not necessarily accurate because the macroanatomical organization of the brain does not always predict where a functional brain area is located (Frost \& Goebel, 2012). Moreover, this approach requires expertise to correctly identify anatomical landmarks and is subjective, thus prone to human error. A second, commonly used, approach is to base TMS target points on coordinates in a common reference space, such as Montreal Neurological Institute (MNI) or Talairach space (Talairach \& Tournoux, 1988). These coordinates are generally based on fMRI group data from prior experiments or taken from the literature. This procedure only requires individual anatomical data, which are transformed into the same reference space to project the Talairach coordinates on them. However, this approach is limited, because Talairach coordinates are represented in volume space, the macroanatomical variability across participants is completely ignored, and the extent of functional-anatomical correspondence of brain areas is not taken into account (Frost \& Goebel, 2012). A third approach makes full use of neuroimaging data by utilizing both individual anatomical and functional data. The target area is functionally defined in each individual participant in a preceding fMRI experiment and targeted directly with neuronavigation. As the research question for the TMS experiment is in most cases whether or not the observed functional activation involves a functionally relevant process, this localization scheme seems most appropriate.

We recently empirically evaluated the impact of these various localization schemes on behavioral effect sizes and statistical power (Sack et al., 2009; also see Sparing et al., 2008). The inferior parietal sulcus was determined by aforementioned localization schemes, and the effects of TMS on task performance were evaluated for each resulting TMS site. We showed that the number of participants required for a TMS effect to reach significance can increase dramatically when localization accuracy is suboptimal. As expected, individual functional localiza- tion of TMS target points based on individual fMRI data outperformed all other available methods. Specifically, TMS effects were significant with just five participants using fMRI-based neuronavigation, whereas neuronavigation based on Talairach coordinates reached the same significance level only after 13 participants.

In summary, localization methods that do not utilize individual functional data for TMS target point localization come at a cost that can be of considerable practical relevance. As shown above, the choice to use individual fMRI-guided TMS can be decisive for finding a significant TMS effect. Yet, the individual functional localization method may not always be feasible or acceptably costefficient. Whereas appropriate functional data may generally be lacking for the recruited subject sample, previously acquired anatomical data are much more widely available. In these cases, traditionally, the Talairach(or MNI-) based approach has been applied as it only requires such anatomical data before TMS. However, these approaches have various shortcomings and demonstrably limited power, as discussed above. Here, we propose a novel method for TMS coil positioning that overcomes some of these shortcomings while still only requiring anatomical data for each participant as well as preexisting functional group data. As will be explained below, we refer to this as the "CBA-based approach" for localization.

In recent years advanced whole-brain alignment schemes have been developed that exploit curvature information of the cortical surface to minimize macroanatomical variability across participants to increase functional overlap on the group level (Frost \& Goebel, 2012; Goebel, Esposito, \& Formisano, 2006; Fischl, Sereno, \& Dale, 1999; Fischl, Sereno, Tootell, \& Dale, 1999). We suggest that these methods can also be used to improve TMS targeting in the absence of individual functional data. To demonstrate, we here use cortex-based alignment (CBA) to transform individual anatomical data to an atlas brain that includes functional probabilistic group maps of the FEF and $\mathrm{hMT}+/ \mathrm{V} 5$. Then, these functional group maps are back-transformed to the individual brain anatomy, preserving functional-anatomical correspondence. These atlas-based functional hotspots can thus serve as TMS target points. As a proof of principle, we measured individual FEF and V5/hMT + hotspots (serving as "baseline") with standard localizers to evaluate the relative locations of TMS target sites based on (1) these individual localizers, (2) our CBA-based approach, and (3) Talairach coordinates. Importantly, this approach can be generalized to any existing fMRI data set in surface space, allowing the use of previously obtained functional results instead of publicly available probabilistic atlases if desired. Given the widespread availability of CBA schemes and preexisting functional group data, the proposed procedure is easy to implement and at no additional measurement costs compared with traditional Talairach- or MNI-based targeting. 


\section{METHODS}

\section{Participants}

Twelve participants (six women; aged 25-31) were recruited from the Maastricht University community. All had normal or corrected-to-normal vision and no history of neurological or psychiatric illness and were screened for fMRI experimentation safety. Written informed consent was obtained before participation, and the study was approved by the ethical committee of the Faculty of Psychology and Neuroscience at Maastricht University.

\section{fMRI}

Anatomical and functional data were acquired with a Siemens Allegra 3.0 Tesla scanner equipped with a standard head coil (Siemens Medical Systems, Erlangen, Germany). High-resolution anatomical images covering the whole head were collected with a T1-weighted 3D ADNI MP-RAGE sequence (192 sagittal slices; matrix $=256 \times$ 256; field of view $=256 \times 256 \mathrm{~mm}$; slice thickness $=1 \mathrm{~mm}$; no gap; in-plane voxel size $=1 \times 1 \mathrm{~mm}$; flip angle $=90^{\circ}$; repetition time $=2250 \mathrm{msec}$; echo time $=2.6 \mathrm{msec}$ ). Functional images were obtained using a T2*-weighted single shot EPI sequence (32 oblique slices with full-brain coverage; matrix $=64 \times 64$; field of view $=224 \times 224 \mathrm{~mm}$; slice thickness $=3.5 \mathrm{~mm}$; no gap; in-plane voxel size $=3.5 \times$ $3.5 \mathrm{~mm}$; flip angle $=90^{\circ}$; repetition time $=2000 \mathrm{msec}$; echo time $=30 \mathrm{msec}$ ). Visual stimuli were back-projected on a screen at the rear end of the scanner bore and could be seen by the participants via a mirror system attached to the head coil. MRI data were analyzed using the BrainVoyager QX 2.4.2 software package (Brain Innovation, Maastricht, The Netherlands), including preprocessing (motion correction, slice scan time correction, spatial smoothing), statistical analysis (general linear model),
Talairach normalization, and CBA. For details, see Frost and Goebel (2012) and Goebel et al. (2006).

\section{Functional Localizers}

We used standard functional localizers to determine the position of FEF and hMT+/V5 in each participant (procedures identical to Frost \& Goebel, 2012). For FEF localization, a block design was used with alternating blocks of central fixation and saccadic eye movements. During saccadic eye movement blocks, participants had to follow a circle with their eyes that appeared at one of eight predefined locations along the horizontal and vertical meridian at a rate of $2 \mathrm{~Hz}$. In total, participants completed 21 blocks each lasting $16 \mathrm{sec}$. The contrast used to identify FEF was "saccades > fixation." For the hMT+/V5 localizer, blocks with a central fixation point were alternated with blocks of stationary or moving "flowfield" dots that were passively viewed. Dot displays consisted of 400 dots that either moved radially outward from the center of the screen or remained stationary throughout the block. Participants were instructed to keep central fixation at all times. In total, participants completed 17 blocks each lasting $16 \mathrm{sec}$. The contrast used to identify hMT+/V5 was "moving dots > stationary dots."

\section{Retrieving FEF and hMT+/V5 TMS Target Points from Independent Group Data}

The central aim of this study was to assess localization precision of TMS target points in cases where individual functional localizers are unavailable (Figure 1). This is conventionally based on coordinates in a standard system, such as Talairach space. To test the efficacy of our CBA-based approach (see below), we used a second and

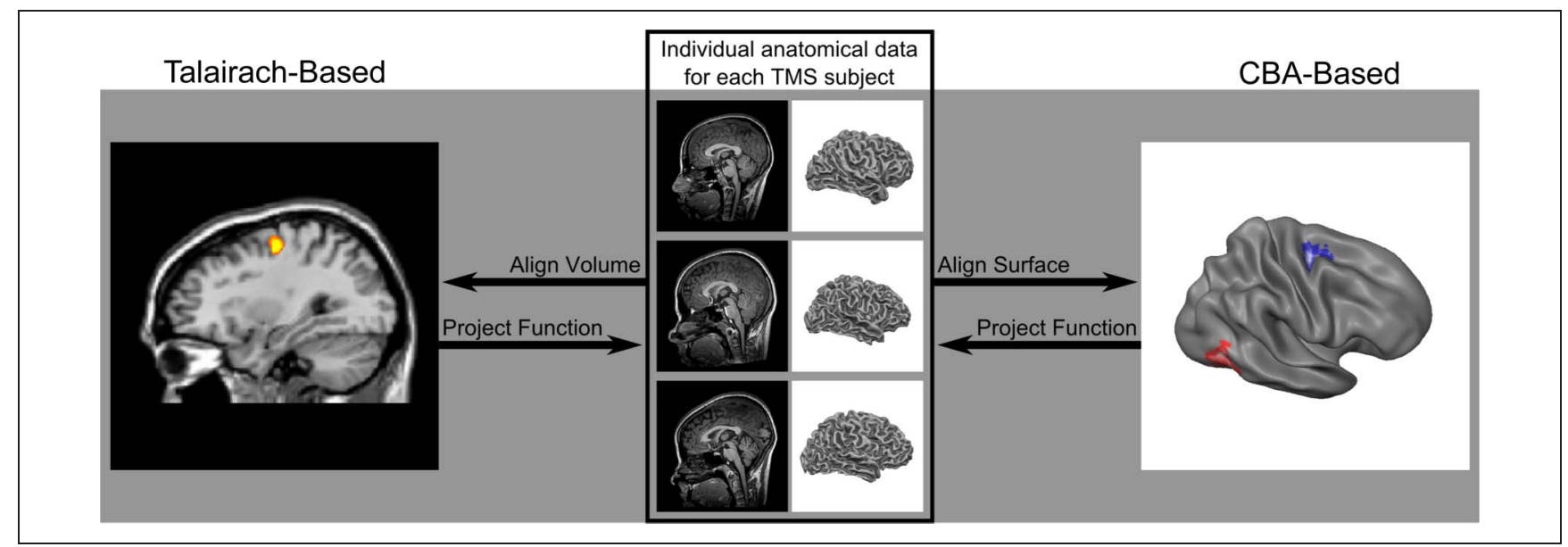

Figure 1. Schematic representation of the traditional Talairach-based approach and our CBA-based approach. Individual anatomical data are aligned to an atlas/reference brain either in volume space based on a few anatomical landmarks, as shown on the left, or in surface space based on the macroanatomical structure of the brain, as shown on the right. Once this alignment is performed, existing functional data can be back-projected to individual space by applying the inverse transformation. 
independent data set from a previous study with other participants (Frost \& Goebel, 2012) to estimate the position of FEF and hMT+/V5 from these group data $(N=$ 10) in each participant. In contrast to most practical situations in TMS research, the localization procedures were actually identical to the ones used for this study (outlined above) to prevent any confounding effects because of task differences. The later comparison between localization methods will therefore be as "clean" as possible. These group data were available in "volume space" where Talairach coordinates defined the location of FEF and hMT+/V5 as they are commonly obtained with a univariate analysis (coordinates representing the center of gravity of a cluster at an individually adjusted threshold) and in "surface space" where probabilistic functional maps indicated the area of highest probability for each cluster (for details, see Frost \& Goebel, 2012). The data represented in surface space were used for our CBAbased approach. For the Talairach-based approach, we projected the independent group Talairach coordinates back to the individual brain anatomy of the participants from this study. For the CBA-based approach, we used the individual anatomical data to create a reconstruction of the cortical surface. Individual anatomical data were then aligned to the average group brain of the independent data set described above using CBA. This alignment process first extracts curvature information from the cortical mesh reconstructions, which are then inflated to a sphere. CBA then aligns in a "coarse to fine" fashion. Initially, alignment uses heavily smoothed curvature maps, which only contain the most prominent macroanatomical landmarks (the central sulcus, the Sylvian fissure, the STS, etc.) to avoid gross misalignment. The next level of alignment uses less smoothed curvature maps and so on until the final stage where alignment proceeds on unsmoothed maps containing all anatomical details. CBA calculates gradient information from curvature maps and utilizes these gradients to iteratively reduce misalignment, through local vertex movement, to a target brain, which here was a previously published atlas brain (see Frost \& Goebel, 2012). Finally, using the inverse of this transformation, the probabilistic functional atlas was back-transformed to the individual brain anatomy where the center of gravity for the area with the highest probability of each cluster (FEF and hMT+/V5) was defined as TMS target point.

\section{Data Analysis}

Following the procedures described above, we obtained three sets of coordinates for each brain area (FEF and hMT+/V5) and participant, namely (1) the individual hotspot based on functional localization, (2) a Talairachbased hotspot obtained from the independent group data set in volume space, and (3) a CBA-based hotspot obtained from the same independent group data set in surface space. Given that individual functional localizers are the gold standard both on theoretical and empirical basis (Sack et al., 2009), they essentially serve as "baselines" for the FEF and hMT+/V5 location in individual participants. Compared with these baselines, the precision of the different localization approaches was then quantified in terms of Euclidean distances between the individually defined hotspots and the Talairach-based and CBA-based hotspots. This measure of distance between hotspots was then used for further statistical analysis.

The performance of Talairach-based and CBA-based targeting was tested with repeated-measures ANOVA with brain area (FEF and hMT+/V5) and localization approach (CBA and Talairach) as within-subject factors. Additionally, we hypothesized that CBA-based targeting is more accurate than localization based on Talairach coordinates and therefore compared both approaches for each brain area directly with one-sided paired $t$ tests.

\section{RESULTS}

\section{Individual Functional Localizers}

We first analyzed the data from the FEF and hMT+/V5 functional localizers on the individual level to determine the precise location of activation foci for all participants. For both tasks, localization was successful in all cases, and individual Talairach coordinates are reported in Table 1.

Table 1. Talairach Coordinates of Individual Functional Hotspots of FEF and hMT+/V5 for Each Participant

\begin{tabular}{|c|c|c|c|c|c|c|}
\hline \multirow[b]{2}{*}{ Participant } & \multicolumn{3}{|c|}{$F E F$} & \multicolumn{3}{|c|}{$b M T+/ V 5$} \\
\hline & $x$ & $y$ & $z$ & $x$ & $y$ & $z$ \\
\hline 1 & 25 & -8 & 46 & 41 & -72 & 5 \\
\hline 2 & 26 & -10 & 58 & 46 & -68 & -2 \\
\hline 3 & 29 & -14 & 56 & 49 & -58 & -6 \\
\hline 4 & 22 & 0 & 54 & 40 & -61 & 5 \\
\hline 5 & 36 & -15 & 55 & 42 & -68 & 3 \\
\hline 6 & 22 & -8 & 53 & 47 & -65 & 4 \\
\hline 7 & 26 & -10 & 51 & 51 & -58 & -2 \\
\hline 8 & 25 & -13 & 46 & 42 & -67 & 2 \\
\hline 9 & 26 & -20 & 53 & 42 & -85 & -7 \\
\hline 10 & 20 & -9 & 54 & 33 & -73 & -4 \\
\hline 11 & 26 & -14 & 53 & 39 & -72 & -4 \\
\hline 12 & 30 & -13 & 44 & 49 & -64 & -8 \\
\hline Mean & 26 & -11 & 52 & 43 & -68 & -1 \\
\hline SEM & 1.2 & 1.4 & 1.3 & 1.5 & 2.2 & 1.4 \\
\hline Range & 16 & 20 & 14 & 18 & 27 & 13 \\
\hline
\end{tabular}

All values are provided in millimeters. Talairach conventions: $x=$ left to right, $y=$ back to front, $z=$ bottom to top. 
These coordinates were considered to represent the "true" locations of the respective areas for each participant that, in the ideal case, would be used as TMS target points. They thus served as reference points that were later used to calculate the relative displacement of TMS target points based on CBA and Talairach coordinates. It is noteworthy that there was a considerable variability across participants. To illustrate, we calculated the range for each dimension of the individual Talairach coordinates as a statistical measure of dispersion. The smallest interval (in millimeters) that included all the data in the $x, y$, and $z$ direction was 16,20 , and 14 for FEF and 18, 27, and 13 for hMT+/V5, respectively. This already shows that a single set of Talairach coordinates is insufficient to accurately predict where a functional brain area is located at the individual level.

To allow comparisons with earlier studies, we also analyzed the data on the group level. As shown in Figure 2, the FEF localizer showed consistent activation near the junction of the precentral sulcus and superior frontal sulcus ( $x=25, y=-10, z=53)$. The hMT+/V5 localizer was found to robustly activate the middle temporal complex ( $x=44, y=-67, z=0$; Figure 2). This closely matches previously reported FEF (e.g., Amiez \& Petrides, 2009; Paus, 1996) and hMT+/V5 (e.g., Tootell et al., 1995) positions and confirms the adequacy of our functional localizers.

\section{CBA versus Talairach Coordinates}

We then assessed the precision of the CBA-based approach and determined the improvement of localization compared with traditional Talairach coordinates. A repeated-measures ANOVA on the Euclidean distance from the individual hotspot was performed with Locali-

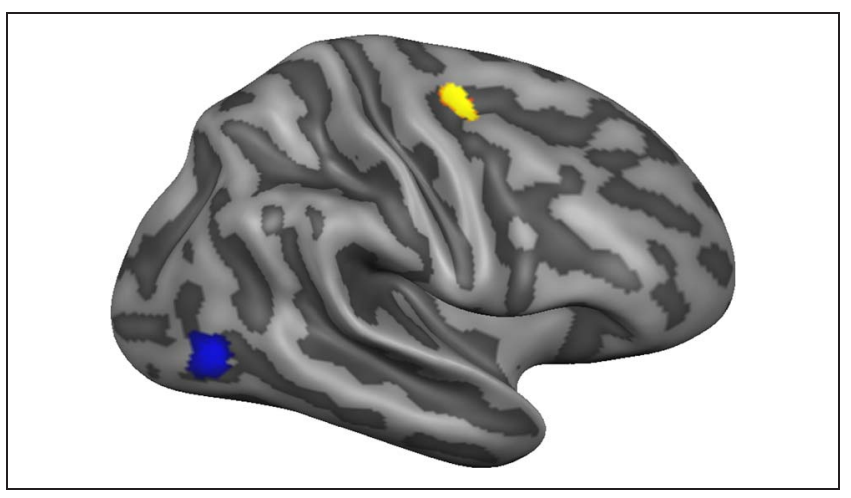

Figure 2. Group activation data projected on the inflated group average brain. The FEF activation hotspot (shown in yellow) was located at the junction of the precentral sulcus and superior frontal sulcus. The contrast to identify FEF was "saccade $>$ fixation." The hMT+/V5 activation hotspot (shown in blue) was located in the middle temporal complex. The contrast used to identify hMT+/V5 was "moving dots > stationary dots." For illustrative purposes, functional data in this figure have been masked so that occipital and parietal activation foci are absent.

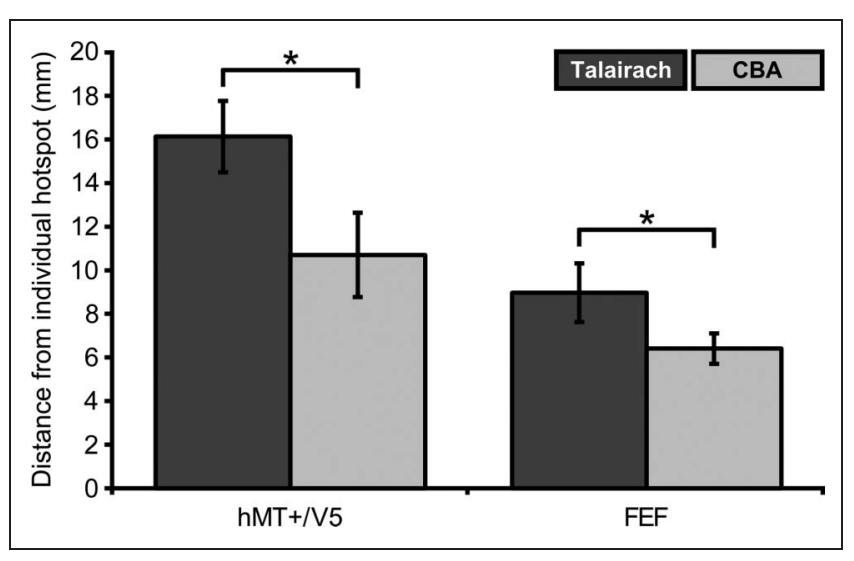

Figure 3. Average localization precision of the CBA-based and Talairach-based approach. Each approach is evaluated by calculating the Euclidean from the individual functional hotspot. For FEF and hMT+/V5, the CBA-based approach outperformed localization based on Talairach coordinates. Differences marked with an asterisk are statistically significant at an alpha level of .05, and error bars depict standard errors.

zation Approach (CBA and Talairach) and Brain Area (FEF and hMT+/V5) as within-subject factors. This revealed a significant main effect of Localization Approach, $F(1,11)=9.580, p=.010$, and Brain Area, $F(1,11)=$ $10.964, p=.007$, but no interaction, $F(1,11)=1.061$, $p=.325$.

The main effect of Localization Approach resulted from higher precision of the CBA-based approach compared with the Talairach-based approach. On average, our approach improved localization by $4.0 \mathrm{~mm}(S E M=1.3)$. Specifically, the mean displacement (in millimeters) of TMS target points based on CBA and Talairach coordinates was 6.4 and 9.0 for FEF and 10.7 and 16.1 for hMT+/V5, respectively (Figure 3). Planned comparisons (pairedsamples $t$ tests, one-tailed) confirmed that these improvements were significant for FEF, $t(11)=1.975, p=.037$, and $\mathrm{hMT}+/ \mathrm{V} 5, t(11)=2.294, p=.021$. The main effect of brain area resulted from generally better localization of FEF compared with hMT+/V5 most likely because of differences in variability across participants as mentioned above.

Because the effectiveness of TMS requires precise localization of TMS target points on the individual level, we further explored the data on a single subject basis. Ideally, the CBA-based approach reduces the distance from the individual hotspot not only on the group level but also in the majority of participants compared with the Talairachbased approach. As shown in Figure 4, individual localization of FEF and hMT+/V5 was improved by our CBA-based approach in 9 of 12 participants.

Finally, we evaluated the distribution of the data in the context of the spatial resolution of TMS. To avoid assuming a specific distance value where TMS is considered to be effective, we created bins with a width of $5 \mathrm{~mm}$ each (representing the distance from the individual hotspots) and counted the number of individual TMS target points 
Figure 4. Localization precision of the CBA-based and Talairach-based approach on the individual level. For FEF (top) and hMT+/V5 (bottom), the CBA-based approach outperformed localization based on Talairach coordinates in 9 of 12 participants. In each plot, participants are sorted, from large to small, according to the distance of the TMS target point based on Talairach coordinates from the individual functional hotspot.

Consequently, there is no systematic correspondence of participant numbers between FEF and hMT+/V5 data.
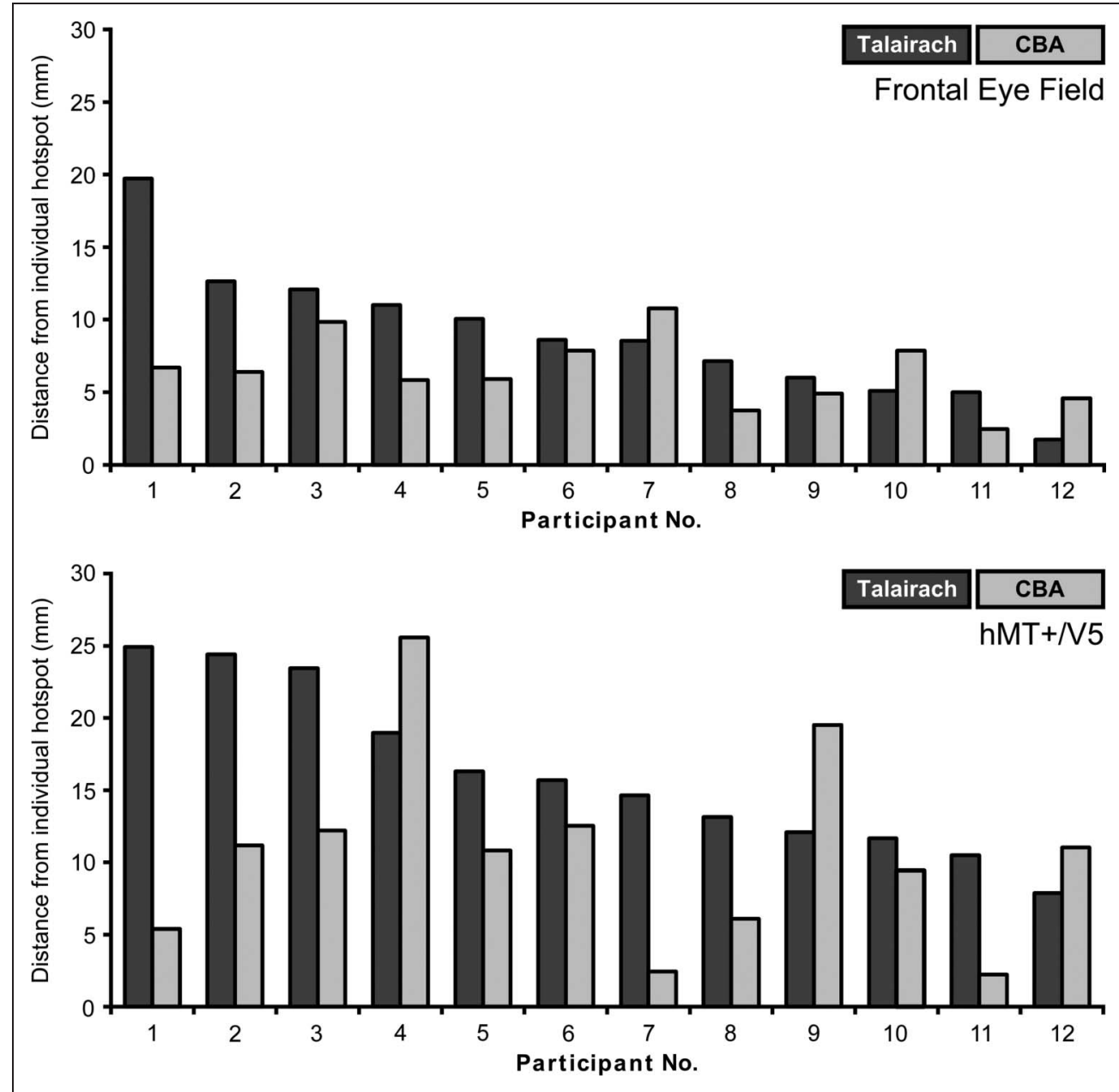

that fell within these intervals. As can be seen in Figure 5, the CBA-based approach not only brought TMS target points closer to the center than the Talairach-based approach on the group level but also pulled a greater number of participants within the effective range of TMS. For example, assuming that the TMS coil should be within $15 \mathrm{~mm}$ from the true location of hMT+/V5 to have an effect, the Talairach-based approach would have been effective in only $50.0 \%$ of the participants whereas the CBA-based approach would have increased this number to $83.3 \%$. Importantly, this advantage of the CBA-based approach was not specific to any particular assumed effective range of TMS.

\section{DISCUSSION}

The success of TMS experiments critically depends on accurate TMS coil positioning. Although functional localization based on individual fMRI data provides best results, we propose a novel method for TMS coil positioning that outperforms alternative approaches, such as targeting based on Talairach coordinates, but still only requires individual anatomical data as well as preexisting

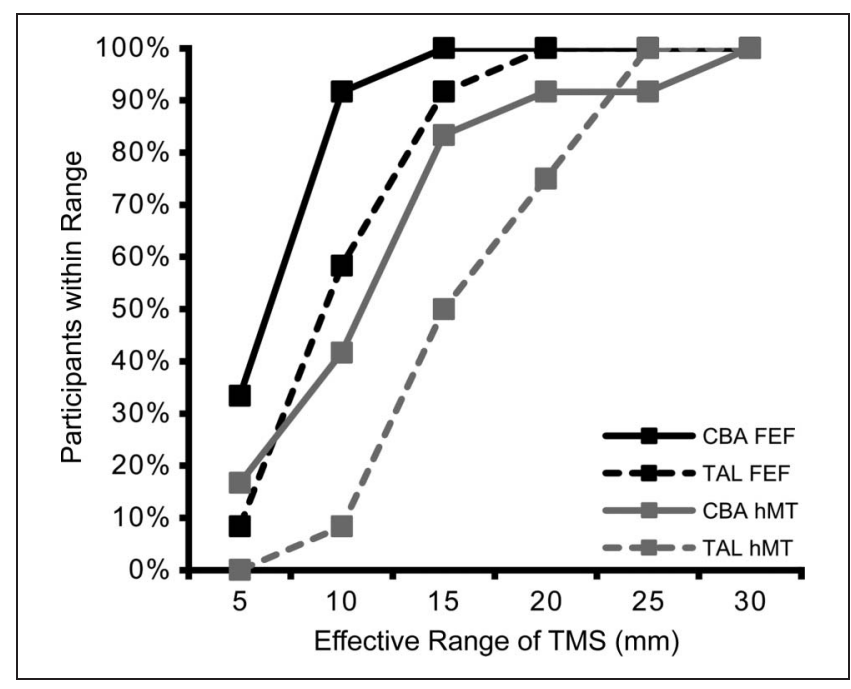

Figure 5. Proportion of participants within the effective range of TMS for each brain area and localization approach. For FEF and hMT+/V5, the CBA-based approach brought more participants within the effective range of TMS compared with targeting based on Talairach coordinates independent of the assumed effective range of TMS. Data are binned at a 5-mm resolution, and the performance of each approach can be evaluated at different cutoff values ranging from 5 to $30 \mathrm{~mm}$. 
functional group data. Our CBA-based approach should therefore be the method of choice either when individual functional data cannot be obtained or experimental factors argue against it (e.g., training or surprise effects).

Our results demonstrate that localization of TMS target points can be significantly improved compared with Talairach-based targeting when utilizing whole-brain alignment schemes that take the macroanatomical differences between participants into account. By incorporating already existing functional group data that are aligned in surface space, the proposed approach takes full advantage of the extent of functional-anatomical correspondence. This is in contrast to localization based on Talairach (or MNI) coordinates where this source of variability is completely ignored and alignment is done in volume space with only few anatomical landmarks serving as reference points. As a side note, we did not evaluate other (nonlinear) volumetric alignment approaches such as SPM DARTEL (Ashburner, 2007) and FSL FNIRT (Andersson, Smith, \& Jenkinson, 2008). To the best of our knowledge, they have not been formally examined in the context of TMS coil positioning, but similar benefits as our CBA-based approach could be expected. A direct comparison of all available nonlinear whole-brain alignment schemes is beyond the scope of the present article. Here, we introduce our CBA-based approach and empirically demonstrate its benefits compared with what is common practice in the TMS community. The greatest advantage of the CBA-based approach is the balance between efficiency and accuracy. It is sufficient to obtain anatomical data for each participant, keeping measurement costs at a minimum (if anatomical data are not present already), whereas localization errors are significantly reduced compared with Talairach-based targeting. The direct comparison of the CBA-based and Talairachbased approach revealed that the CBA-based approach performed better in the majority of cases with improvements of a few millimeters on average. As will be discussed below, even these relatively small differences can be of practical relevance in the context of a TMS experiment.

Previous work strongly suggests that the difference in accuracy between the CBA-based and Talairach-based approach reported here would translate into differences in effect size and statistical power. Sack et al. (2009) directly compared TMS-induced changes in task behavior using different approaches for TMS coil positioning, namely targeting based on (1) individual functional localizer, (2) individual brain anatomy, (3) Talairach coordinates, and (4) EEG electrode positions. Results showed that the number of participants required for a TMS effect to reach significance, as well as the risk of false negatives (Type II error), can increase dramatically when localization accuracy is suboptimal. On the basis of this statistical argument, it is recommended to always use the best TMS coil positioning approach available. Moreover, previous work has shown that spatial displacements of the TMS coil in the order of millimeters can abolish behavioral effects altogether (Sack et al., 2006). Given that the strength of the magnetic field produced by the TMS coil decreases nonlinearly with distance, such abrupt changes in TMS efficacy are hardly surprising. For the same reason, it remains an open empirical question to what extent the improvements of our CBA-based approach compared with the Talairach-based approach translate into differences in TMS effects in the context of a given task and stimulation site. Still, bringing the TMS coil as close as possible to the brain area of interest should always be a priority in TMS experiments, and we therefore argue that the CBA-based approach should be the method of choice when individual functional localization is impossible.

The widespread use of Talairach space in neuroimaging has certainly contributed to the popularity of Talairach coordinates in TMS coil positioning. Transforming individual data to a common space is a crucial prerequisite for most group analyses and, importantly, allows comparisons across experiments. Group activation data can be reduced to a simple set of coordinates, which also allows their easy use in a separate TMS experiment. However, the limitations of data representation in Talairach space have long been recognized, and advanced whole-brain alignment schemes are more and more used in the neuroimaging community. In light of these developments, we propose that the TMS community should follow this development and take advantage of these improvements in the context of TMS coil positioning, as we demonstrate here.

We used a recently developed functional probabilistic atlas for this study (Frost \& Goebel, 2012). This atlas contains functional probabilistic group maps of 10 brain areas and may be obtained by contacting the corresponding author but will soon be publicly available as well. By creating a probabilistic atlas, one is able to identify the degree to which functional areas "respect" macroanatomical landmarks. Functional areas that are strongly bound to macroanatomy are represented in the atlas as a small patch of cortical surface where there is a high likelihood that any new participant's functional area would be in the same anatomical location. By aligning a brain to this atlas brain, these probabilities can be used in participants for whom functional data are not available. One must note however that these maps only represent the probabilistic location of the functional area. Although they are derived from functional data one cannot say, with complete certainty, that a particular functional area will occupy the same patch of cortex in all.

Finally, although the CBA-based approach improves TMS coil positioning compared with Talairach-based targeting, our results also show that localization is still far from perfect. It is important to realize that this remaining localization error may still be decisive for finding a significant TMS effect in a given participant or patient. This remaining localization error of the CBA approach is brought about by a couple of general limitations that are inherent to any approach not using individual functional data and that can also not be overcome 
by using the here proposed CBA-based approach. First, the core of our approach is to improve localization of functional brain areas by incorporating macroanatomical information. This obviously requires some degree of functional-anatomical correspondence. If the location of any given functional brain area is in no way related to the available anatomical data, then it is impossible to make any predictions based on anatomy alone. In such cases, nothing but individual functional localizers will be successful. Second, there is always the possibility of idiosyncrasies, that is, participants with functional brain areas that do not conform to regularities found in brain anatomy on the group level. This obviously cannot be captured with our approach, as these cases will be poorly localized, even with anatomical alignment. Again, nothing but individual functional localizers will be successful.

In conclusion, we propose a novel approach of TMS coil positioning that significantly improves localization accuracy compared with alternative approaches in situations where individual functional localization is not possible but preexisting functional group data are available. We demonstrated the benefits of accounting for macroanatomical variability across participants for two brain areas, namely FEF and hMT+/V5. Consequently, we suggest that the TMS community could embrace the development of whole-brain alignment schemes from the field of neuroimaging to improve TMS coil positioning. Finally, it is noteworthy that the CBA-based approach is not limited to TMS experiments. If the precision of the CBAbased approach is considered necessary and sufficient, it might be useful for other applications where targeting of functional brain areas is required, for example, in optical imaging, electrode placement, or the like.

\section{Acknowledgments}

This work was supported by the European Research Council under the European Union's Seventh Framework Programme (FP7/2007-2013)/ERC Grant Agreement (263472, A. T. S. and 269853, R. G.) and the Netherlands Organization for Scientific Research (400-07-230, A. T. S., and 451-13-024, T. A. G.).

Reprint requests should be sent to Felix Duecker, Department of Cognitive Neuroscience, Faculty of Psychology and Neuroscience, Maastricht University, PO Box 616, 6200 MD Maastricht, The Netherlands, or via e-mail: felix.duecker@maastrichtuniversity.nl.

\section{REFERENCES}

Amiez, C., \& Petrides, M. (2009). Anatomical organization of the eye fields in the human and non-human primate frontal cortex. Progress in Neurobiology, 89, 220-230.

Andersson, J. L. R., Smith, S. M., \& Jenkinson, M. (2008). FNIRTFMRIB's non-linear image registration tool. Proceedings of the 14th Annual Meeting of the Organization for Human Brain Mapping. Neuroimage, 41.

Ashburner, J. (2007). A fast diffeomorphic image registration algorithm. Neuroimage, 38, 95-113.

Cohen Kadosh, R., Cohen Kadosh, K., Schuhmann, T., Kaas, A., Goebel, R., Henik, A., et al. (2007). Virtual dyscalculia induced by parietal-lobe TMS impairs automatic magnitude processing. Current Biology, 17, 689-693.

Deng, Z.-D., Lisanby, S. H., \& Peterchev, A. V. (2012). Electric field depth-focality tradeoff in transcranial magnetic stimulation: Simulation comparison of 50 coil designs Brain Stimulation, 6, 1-13.

Fischl, B., Sereno, M. I., \& Dale, A. M. (1999). Cortical surfacebased analysis II: Inflation, flattening, and a surface-based coordinate system. Neuroimage, 9, 195-207.

Fischl, B., Sereno, M. I., Tootell, R. B., \& Dale, A. M. (1999). High-resolution intersubject averaging and a coordinate system for the cortical surface. Human Brain Mapping, 8, 272-284.

Frost, M. A., \& Goebel, R. (2012). Measuring structuralfunctional correspondence: Spatial variability of specialised brain regions after macro-anatomical alignment. Neuroimage, 59, 1369-1381.

Goebel, R., Esposito, F., \& Formisano, E. (2006). Analysis of functional image analysis contest (FIAC) data with brainvoyager QX: From single-subject to cortically aligned group general linear model analysis and self-organizing group independent component analysis. Human Brain Mapping, 27, 392-401.

Hallett, M. (2000). Transcranial magnetic stimulation and the human brain. Nature, 406, 147-150.

Herwig, U., Kölbel, K., Wunderlich, A. P., Thielscher, A., Von Tiesenhausen, C., Spitzer, M., et al. (2002). Spatial congruence of neuronavigated transcranial magnetic stimulation and functional neuroimaging. Clinical Neurophysiology, 113, 462-468.

Herwig, U., Satrapi, P., \& Schönfeldt-Lecuona, C. (2003). Using the international 10-20 EEG system for positioning of transcranial magnetic stimulation. Brain Topography, 16, 95-99.

Jasper, H. H. (1958). The ten-twenty electrode system of the International Federation. Electroencephalography and Clinical Neurophysiology, 10, 371-375.

Kammer, T., Puls, K., Erb, M., \& Grodd, W. (2005). Transcranial magnetic stimulation in the visual system. II. Characterization of induced phosphenes and scotomas. Experimental Brain Research, 160, 129-140.

Pascual-Leone, A., Walsh, V., \& Rothwell, J. (2000). Transcranial magnetic stimulation in cognitive neuroscience-Virtual lesion, chronometry, and functional connectivity. Current Opinion in Neurobiology, 10, 232-237.

Paus, T. (1996). Location and function of the human frontal eye-field: A selective review. Neuropsychologia, 34, 475-483.

Paus, T. (1999). Imaging the brain before, during, and after transcranial magnetic stimulation. Neuropsychologia, 37, 219-224.

Sack, A. T. (2006). Transcranial magnetic stimulation, causal structure-function mapping and networks of functional relevance. Current Opinion in Neurobiology, 16, 593-599.

Sack, A. T., Cohen Kadosh, R., Schuhmann, T., Moerel, M., Walsh, V., \& Goebel, R. (2009). Optimizing functional accuracy of TMS in cognitive studies: A comparison of methods. Journal of Cognitive Neuroscience, 21, 207-221.

Sack, A. T., Kohler, A., Linden, D. E. J., Goebel, R., \& Muckli, L. (2006). The temporal characteristics of motion processing in hMT/V5+: Combining fMRI and neuronavigated TMS. Neuroimage, 29, 1326-1335.

Salminen-Vaparanta, N., Noreika, V., Revonsuo, A., Koivisto, M., \& Vanni, S. (2012). Is selective primary visual cortex stimulation achievable with TMS? Human Brain Mapping, 33, 652-665. 
Schönfeldt-Lecuona, C., Thielscher, A., Freudenmann, R. W., Kron, M., Spitzer, M., \& Herwig, U. (2005). Accuracy of stereotaxic positioning of transcranial magnetic stimulation. Brain Topography, 17, 253-259.

Sparing, R., Buelte, D., Meister, I. G., Paus, T., \& Fink, G. R. (2008). Transcranial magnetic stimulation and the challenge of coil placement: A comparison of conventional and stereotaxic neuronavigational strategies. Human Brain Mapping, 29, 82-96.

Talairach, J., \& Tournoux, P. (1988). Co-planar stereotaxic atlas of the buman brain. New York: Thieme.

Thielscher, A., Reichenbach, A., Uğurbil, K., \& Uludağ, K. (2010). The cortical site of visual suppression by transcranial magnetic stimulation. Cerebral Cortex, 20, 328-338.
Thompson, B., Aaen-Stockdale, C., Koski, L., \& Hess, R. F. (2009). A double dissociation between striate and extrastriate visual cortex for pattern motion perception revealed using rTMS. Human Brain Mapping, 30, 3115-3126.

Tootell, R. B., Reppas, J. B., Kwong, K. K., Malach, R., Born, R. T., Brady, T. J., et al. (1995). Functional analysis of human MT and related visual cortical areas using magnetic resonance imaging. The Journal of Neuroscience, 15, 3215-3230.

Walsh, V., \& Cowey, A. (2000). Transcranial magnetic stimulation and cognitive neuroscience. Nature Reviews Neuroscience, 1, 73-79.

Wassermann, E. M., Mcshane, L. M., Hallett, M., \& Cohen, L. G. (1992). Noninvasive mapping of muscle representations in human motor cortex. Electroencephalography and Clinical Neurophysiology, 85, 1-8. 